\title{
A Posteriori Error Bounds for Numerical Solutions of the Neutron Transport Equation
}

\author{
By Niel K. Madsen
}

\begin{abstract}
The theory and application of a method for computing rigorous a posteriori error bounds for numerical solutions to time-independent neutron transport problems are presented. The bounds are obtained for the $L_{2}$ and $L_{1}$ norms of the error function.
\end{abstract}

Introduction. When an approximate numerical solution to a neutron transport problem is computed, it is usually not known just how accurate the solution may be. Therefore, it would be useful to have a computational procedure which would yield bounds on the errors introduced by the numerical approximation. In this paper, a method is presented which gives a posteriori error bounds for numerical approximations to the time-independent one-velocity linear Boltzmann equation in rectangular geometry. The bounds are obtained for the $L_{2}$ and $L_{1}$ norms of the error. Numerical results are also presented.

Formulation. Let the domain $D=R \times S$ be defined by

$$
\begin{aligned}
& R=\left\{\mathbf{x}=\left(x_{1}, x_{2}, x_{3}\right): 0 \leqq x_{1} \leqq a_{1}, 0 \leqq x_{2} \leqq a_{2}, 0 \leqq x_{3} \leqq a_{3}\right\}, \\
& \mathcal{S}=\left\{\mathbf{\Omega}=\left(\Omega_{1}, \Omega_{2}, \Omega_{3}\right): \Omega_{1}^{2}+\Omega_{2}^{2}+\Omega_{3}^{2}=1\right\} .
\end{aligned}
$$

The neutron transport problem considered in this paper is to find a function $\phi$ defined on $D$ which satisfies the time-independent one-velocity linear Boltzmann equation

$$
\mathbf{\Omega} \cdot \nabla_{\mathbf{x}} \phi(\mathbf{x}, \mathbf{\Omega})+\Sigma^{T}(\mathbf{x}) \phi(\mathbf{x}, \mathbf{\Omega})-\int_{S} \Sigma^{S}\left(\mathbf{x}, \mathbf{\Omega}, \mathbf{\Omega}^{\prime}\right) \phi\left(\mathbf{x}, \mathbf{\Omega}^{\prime}\right) d \mathbf{\Omega}^{\prime}=S(\mathbf{x}, \mathbf{\Omega})
$$

where

$\nabla_{\mathrm{x}}$ denotes the gradient with respect to the variable $\mathbf{x}$,

$\Sigma^{T}(\mathbf{x})$, the total cross section at $\mathbf{x}$, is a given bounded positive function which is bounded away from zero,

$\Sigma^{S}\left(\mathbf{x}, \boldsymbol{\Omega}, \boldsymbol{\Omega}^{\prime}\right)$, the differential production cross section from scattering or fission, is a given nonnegative function on $D \times S$ which satisfies $\int_{\delta} \Sigma^{S}\left(\mathbf{x}, \mathbf{\Omega}, \mathbf{\Omega}^{\prime}\right) d \mathbf{\Omega}^{\prime}<\Sigma^{T}(\mathbf{x})$ for all $\mathbf{x} \in R$ and $\boldsymbol{\Omega} \in \mathcal{S}$,

$S(\mathrm{x}, \mathbf{\Omega})$, the distributed neutron source density, is a given bounded function on $D$.

The unknown function $\phi(x, \Omega)$ is called the directional neutron flux at the point $\mathbf{x}$ in the direction $\mathbf{\Omega}$.

Received November 28, 1972.

AMS (MOS) subject classifications (1970). Primary 65N15, 65R05; Secondary 35B30, 35L50, $35 \mathrm{Q} 99$.

Key words and phrases. A posteriori error bounds, neutron transport equation, linear Boltzmann equation, computable error bounds, $L_{2}$ error bounds, $L_{1}$ error bounds, $x-y$ geometry. 
The solution of (1) will be subject to certain conditions to be specified on $\partial R$, the boundary of $R$. Several different types of boundary conditions are frequently specified: vacuum, reflecting, periodic, etc. To be more concise, only vacuum boundary conditions are explicitly considered in this paper. However, all of the results presented are valid for reflecting and periodic boundary conditions (the same analysis techniques apply). The vacuum boundary conditions considered are given by

$$
\phi(\mathbf{x}, \boldsymbol{\Omega})=0 \text { for } \mathbf{x} \in \partial R \text { and } \boldsymbol{\Omega} \text { such that } \boldsymbol{\Omega} \cdot \mathbf{n}<0,
$$

where $\mathbf{n}$ is an outward drawn normal at $\mathbf{x}$.

By a solution $\phi$ of the problem (1), (2), it is meant that $\phi(x+s \Omega, \Omega)$ is an absolutely continuous function of $s$ for almost every $(\mathrm{x}, \boldsymbol{\Omega}) \in \mathscr{D}$, and $\phi$ satisfies Eqs. (1) and (2) almost everywhere in $D$.

This general formulation of the problem has been chosen because it is well known ([1], [4], [6]) that even with arbitrarily smooth coefficient functions, the solution of the transport problem can have singularities in its first derivatives.

Under essentially the above stated conditions, Vladimirov [6] has proved the existence and uniqueness of a solution to the problem (1), (2) provided that $S(\mathrm{x}, \mathbf{\Omega}) \in$ $L_{2}(D)$. The solution $\phi$ is also shown to be in $L_{2}(D)$. Similar results for the transport problem with reflecting and periodic boundary conditions have been established by Kellogg [3].

A Fundamental Inequality. In this section, an inequality is developed which provides the mathematical basis for the a posteriori error bounds procedure. It is convenient to define the following function:

$$
\Sigma^{a}(\mathbf{x}, \mathbf{\Omega}) \equiv \Sigma^{T}(\mathbf{x})-\int_{S} \Sigma^{S}\left(\mathbf{x}, \mathbf{\Omega}, \mathbf{\Omega}^{\prime}\right) d \mathbf{\Omega}^{\prime} .
$$

The following additional assumptions are made:

$$
\begin{aligned}
\Sigma^{a}(\mathbf{x}, \boldsymbol{\Omega}) & \geqq \Sigma_{0}>0 & & \text { for almost all }(\mathbf{x}, \boldsymbol{\Omega}) \in \mathscr{D}, \\
\Sigma^{S}\left(\mathbf{x}, \boldsymbol{\Omega}, \boldsymbol{\Omega}^{\prime}\right) & =\Sigma^{S}\left(\mathbf{x}, \boldsymbol{\Omega}^{\prime}, \boldsymbol{\Omega}\right) & & \text { for almost all }\left(\mathbf{x}, \boldsymbol{\Omega}, \mathbf{\Omega}^{\prime}\right) \in \mathscr{D} \times \mathcal{S} .
\end{aligned}
$$

With the preceding definitions and assumptions the fundamental inequality can now be established.

THEOREM. If $\phi$ is the solution to a transport problem where $S \in L_{2}(D)$, then

$$
\|\phi\|_{L_{2}} \leqq \frac{1}{\Sigma_{0}}\|S\|_{L_{2}} .
$$

Proof. When Eq. (1) is multiplied by $\phi$ and integrated over $D$, it becomes

$$
\begin{aligned}
\frac{1}{2} \int_{\mathscr{D}} \mathbf{\Omega} & \cdot \nabla_{\mathbf{x}} \phi^{2} d \mathbf{x} d \mathbf{\Omega} \\
& +\int_{\mathscr{D}} \Sigma^{T} \phi^{2} d \mathbf{x} d \mathbf{\Omega} \\
& -\int_{\mathcal{D}} \phi(\mathbf{x}, \mathbf{\Omega})\left[\int_{S} \Sigma^{S}\left(\mathbf{x}, \mathbf{\Omega}, \boldsymbol{\Omega}^{\prime}\right) \phi\left(\mathbf{x}, \mathbf{\Omega}^{\prime}\right) d \mathbf{\Omega}^{\prime}\right] d \mathbf{x} d \mathbf{\Omega}=\int_{\mathbb{D}} \phi S d \mathbf{x} d \mathbf{\Omega}
\end{aligned}
$$


Using Fubini's theorem, Green's theorem and the boundary condition (2), it is easily seen that the first integral appearing in (6) is nonnegative. Using (3) and Fubini's theorem, the second two integrals of (6) may be expressed as

$$
\begin{aligned}
\int_{\mathbb{D}} \Sigma^{a} \phi^{2} d \mathbf{x} d \mathbf{\Omega}+\int_{\mathcal{D} \times S}\left[\Sigma^{S}\left(\mathbf{x}, \mathbf{\Omega}, \mathbf{\Omega}^{\prime}\right) \phi^{2}(\mathbf{x}, \mathbf{\Omega})\right. \\
\left.-\Sigma^{S}\left(\mathbf{x}, \mathbf{\Omega}, \mathbf{\Omega}^{\prime}\right) \phi(\mathbf{x}, \mathbf{\Omega}) \phi\left(\mathbf{x}, \mathbf{\Omega}^{\prime}\right)\right] d \mathbf{x} d \mathbf{\Omega} d \mathbf{\Omega}^{\prime} .
\end{aligned}
$$

The goal now is to show that the second integral in (7) is nonnegative. To accomplish this, (5) is now used to give the equality

$$
\begin{aligned}
\int_{\mathfrak{D} \times} \Sigma^{S}\left(\mathbf{x}, \mathbf{\Omega}, \mathbf{\Omega}^{\prime}\right) \phi^{2}(\mathbf{x}, \mathbf{\Omega}) d \mathbf{x} d \mathbf{\Omega} d \mathbf{\Omega}^{\prime} \\
=\frac{1}{2} \int_{\mathbb{D} \times S} \Sigma^{S}\left(\mathbf{x}, \mathbf{\Omega}, \mathbf{\Omega}^{\prime}\right) \phi^{2}(\mathbf{x}, \mathbf{\Omega}) d \mathbf{x} d \mathbf{\Omega} d \mathbf{\Omega}^{\prime} \\
+\frac{1}{2} \int_{\mathbb{D} \times \mathcal{S}} \Sigma^{S}\left(\mathbf{x}, \mathbf{\Omega}, \mathbf{\Omega}^{\prime}\right) \phi^{2}\left(\mathbf{x}, \mathbf{\Omega}^{\prime}\right) d \mathbf{x} d \mathbf{\Omega} d \mathbf{\Omega}^{\prime}
\end{aligned}
$$

Using (8), expression (7) may be written as

$$
\int_{\mathbb{D}} \Sigma^{a} \phi^{2} d \mathbf{x} d \mathbf{\Omega}+\frac{1}{2} \int_{D \times S} \Sigma^{S}\left(\mathbf{x}, \mathbf{\Omega}, \Omega^{\prime}\right)\left[\phi(\mathbf{x}, \mathbf{\Omega})-\phi\left(\mathbf{x}, \mathbf{\Omega}^{\prime}\right)\right]^{2} d \mathbf{x} d \mathbf{\Omega} d \mathbf{\Omega}^{\prime}
$$

and so the second integral of (7) is nonnegative. Therefore, from (4), (6), (7), (9) and Schwarz's inequality, it is seen that

$$
\Sigma_{0}\|\phi\|_{L_{2}}^{2} \leqq \int_{D}|\phi S| d \mathbf{x} d \Omega \leqq\|\phi\|_{L_{2}}\|S\|_{L_{2}}
$$

and the inequality is established.

An inequality involving the $L_{1}$ norm of $\phi$ can now be established as a corollary to the preceding theorem.

COROLlARY. If $\phi$ is the solution to a transport problem where $S \in L_{2}(D)$, then

$$
\|\phi\|_{L_{1}} \leqq \frac{(4 \pi V)^{1 / 2}}{\Sigma_{0}}\|S\|_{L_{2}}
$$

where $V$ is the volume of the domain $R$.

Proof.

$$
\begin{aligned}
\|\phi\|_{L_{1}} & =\int_{\mathcal{D}}|\phi| d \mathbf{x} d \mathbf{\Omega} \leqq\left[\int_{\mathcal{D}} d \mathbf{x} d \mathbf{\Omega}\right]^{1 / 2} \cdot\left[\int_{\mathbb{D}} \phi^{2} d \mathbf{x} d \mathbf{\Omega}\right]^{1 / 2} \\
& =(4 \pi V)^{1 / 2}\|\phi\|_{L_{2}} \leqq \frac{(4 \pi V)^{1 / 2}}{\Sigma_{0}}\|S\|_{L_{2}} .
\end{aligned}
$$

These inequalities can now be used to compute a posteriori error bounds for a given approximation to the solution of a transport problem. For convenience, let $L$ denote the neutron transport operator so that $L \phi=S$ represents Eq. (1). If $\phi$ is a known approximation to the actual solution $\phi$, then the error in the approximation is defined as $e=\phi-\phi$.

THEOREM. Let the given approximate transport solution $\phi(\mathrm{x}+s \mathbf{\Omega}, \mathbf{\Omega})$ be an absolutely continuous function of $s$ for almost every $(\mathbf{x}, \mathbf{\Omega}) \in \mathscr{D}$ such that $\phi \in L_{2}(D)$ 
and $L \Phi \in L_{2}(D)$. If $\Phi$ satisfies (2) almost everywhere then

$$
\|e\|_{L_{2}} \leqq \frac{1}{\Sigma_{0}}\|S-L \tilde{\phi}\|_{L_{2}} \text { and }\|e\|_{L_{1}} \leqq \frac{(4 \pi V)^{1 / 2}}{\Sigma_{0}}\|S-L \tilde{\phi}\|_{L_{2}}
$$

Proof. Applying the transport operator to the error function, it is seen that

$$
L e=L(\phi-\Phi)=L \phi-L \Phi=S-L \tilde{\phi},
$$

that is, $e$ is the solution to a transport problem where the source is now $S-L \Phi$. The desired bounds now follow easily from the inequalities already developed. Since $S$ and $\phi$ are assumed given, the function $S-L \phi$ is known and its $L_{2}$ norm can be computed.

Practical Implementation. There are several considerations which must be made in order to implement the above theorems into a practical computational procedure. First, when an approximate solution to a transport problem is obtained, it is usually in the form of discrete values at mesh points $\left(x_{i}, y_{j}\right)$ and only for certain chosen directions $\boldsymbol{\Omega}^{m}$. Therefore, some type of "smoothing" process must be specified in order to give an approximate solution $\phi$ of the form required. Second, for ease of computing integrals and derivatives, the form of $\phi$ must be relatively simple. Finally, most approximate solutions for transport problems in rectangular geometry are obtained for domains $R$ of dimension one or two. So, the theory presented here for $R$ of dimension three must be applied in practice for lower dimensions (the same proofs appropriately reduced to lower dimensions apply, so everything remains valid).

Assume then that $R$ is of dimension two and that discrete approximate solution values $\phi_{i j}^{m}$ have been obtained by using, for example, a finite difference approximation to the solution of the discrete ordinate equations [2] which approximate the transport equation. It is assumed that a rectangular mesh (not necessarily uniform) has been imposed on $R$. Also, $\delta$, which may be visualized as the surface of the unit sphere, is assumed to be partitioned into a finite number of segments $A^{m}$, each being represented by a particular direction $\boldsymbol{\Omega}^{m} \in S$ and having an area of $w^{m}, m=1,2, \cdots, N$ (see Fig. 1).

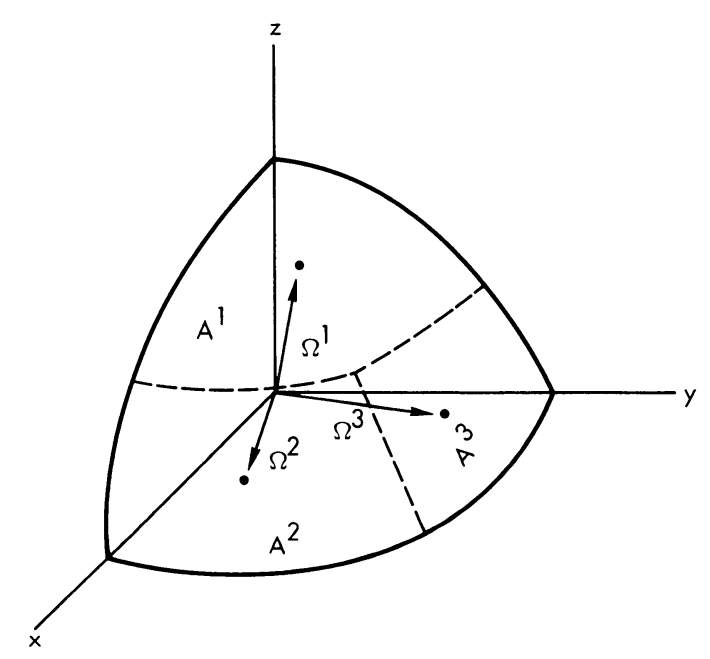

Figure 1. Partitioning of One Octant of the Unit Sphere 


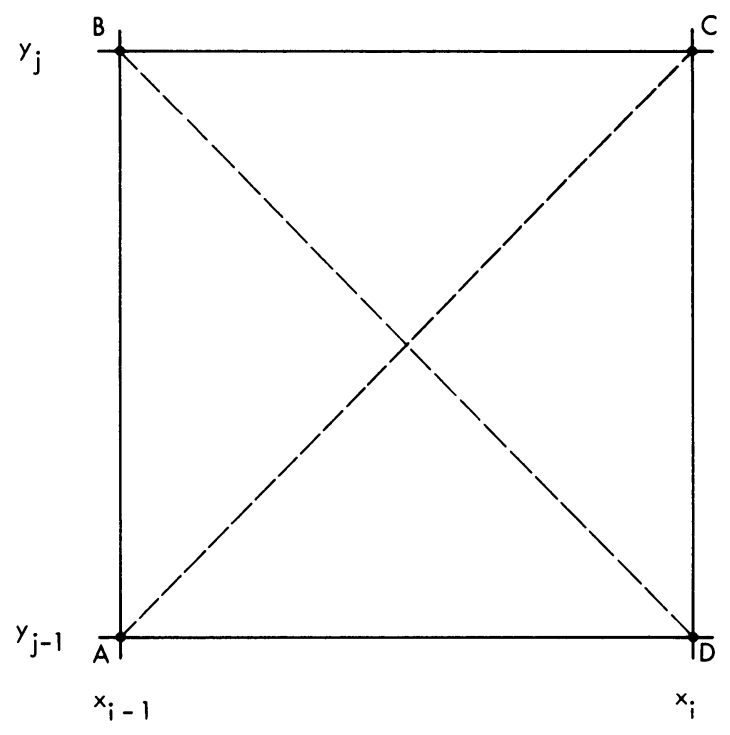

FIGURE 2

The discrete values $\phi_{i j}^{m}$ are used to define an approximate solution $\phi$ over all of $\mathcal{D}$ in the following manner.

Consider a particular mesh box as shown in Fig. 2. If the projection of $\mathbf{\Omega}^{m}$ onto the $x-y$ plane lies in quadrants one or three (i.e., $\Omega_{1}^{m} \Omega_{2}^{m} \geqq 0$ ), then a planar function $\Phi^{m}=a+b x+c y$ is defined over each of the triangles $A C D$ and $A B C$ using the known discrete values at the vertices.

If the projection of $\boldsymbol{\Omega}^{m}$ lies in quadrants two or four (i.e., $\Omega_{1}^{m} \Omega_{2}^{m}<0$ ), then a similar planar function $\phi^{m}$ is defined over the triangles $A B D$ and $B C D$. The final approximate solution $\Phi$ is obtained by extending the functions $\phi^{m}$ to have values for all $\boldsymbol{\Omega} \in A^{m}$, that is, $\phi(x, y, \mathbf{\Omega})=\phi^{m}(x, y)$ if $\boldsymbol{\Omega} \in A^{m}$. Therefore, for each given point $(x, y) \in R, \phi$ is a piecewise constant function of $\Omega$. The values $\phi$ assumes on the boundaries of each $A^{m}$ are not of real concern as these boundaries are a set of measure zero in $S$.

From the manner in which $\phi$ has been defined, it is evident that $\phi(x+s P \Omega, \Omega)$ ( $P \Omega$ is the projection of $\Omega$ onto the $x-y$ plane) is an absolutely continuous function of $s$ for almost every $(\mathbf{x}, \Omega) \in D$ and satisfies the boundary conditions (2) everywhere. Also, because of the simple form of the approximate solution, integrals and derivatives of $\phi$ can be easily and explicitly computed. For example, the integral $\int_{\delta} \phi(x, y, \boldsymbol{\Omega}) d \boldsymbol{\Omega}$ is easily seen to be $\sum_{m=1}^{N} w^{m} \hat{\phi}^{m}(x, y)$. In actual applications, the functions $S, \Sigma^{T}$, and $\Sigma^{S}$ are usually piecewise constant, so that the error bound $\|S-L \Phi\|_{L_{3}} / \Sigma_{0}$ can be computed without difficulty.

It should be mentioned that the "smoothing" procedure used to obtain $\phi$ is quite arbitrary and the above method was chosen largely because of its simplicity and also because it seemed to give better bounds than other simple methods tried.

Results. A computer program which actually computes the approximate solution $\Phi$ and then the $L_{1}$ and $L_{2}$ error bounds was written and tested for a number of problems. The results obtained for three particular problems are presented. The first problem 


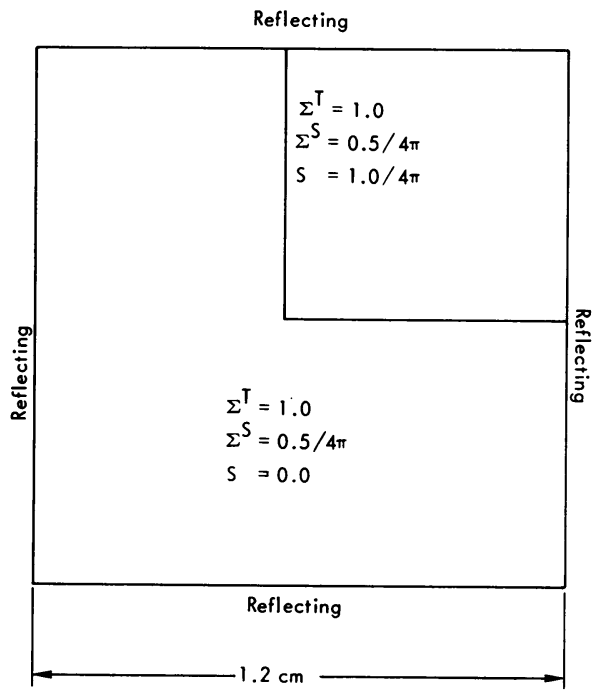

FIGURE 3

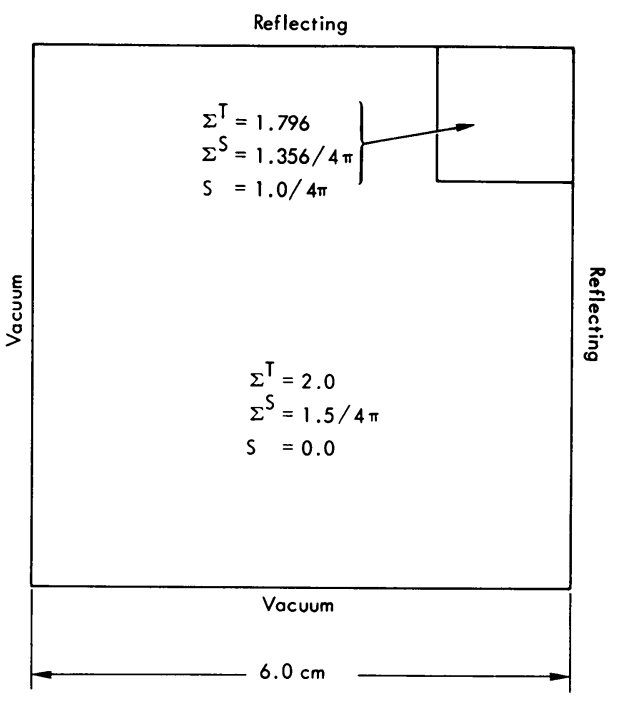

FIGURE 4

(see Fig. 3) is a model cell problem with four reflecting boundaries and a rectangular source area located in one corner. The second problem (see Fig. 4) is a model shielding problem with two reflecting boundaries and two vacuum boundaries and a source in the corner formed by the reflecting boundaries. The third problem (see Fig. 5) is a more practical type of problem. It comes from an idealization of a seed-blanket cell for a pressurized water reactor [5].

The $L_{2}$ error bounds for the three problems are shown in Table 1 and the $L_{1}$

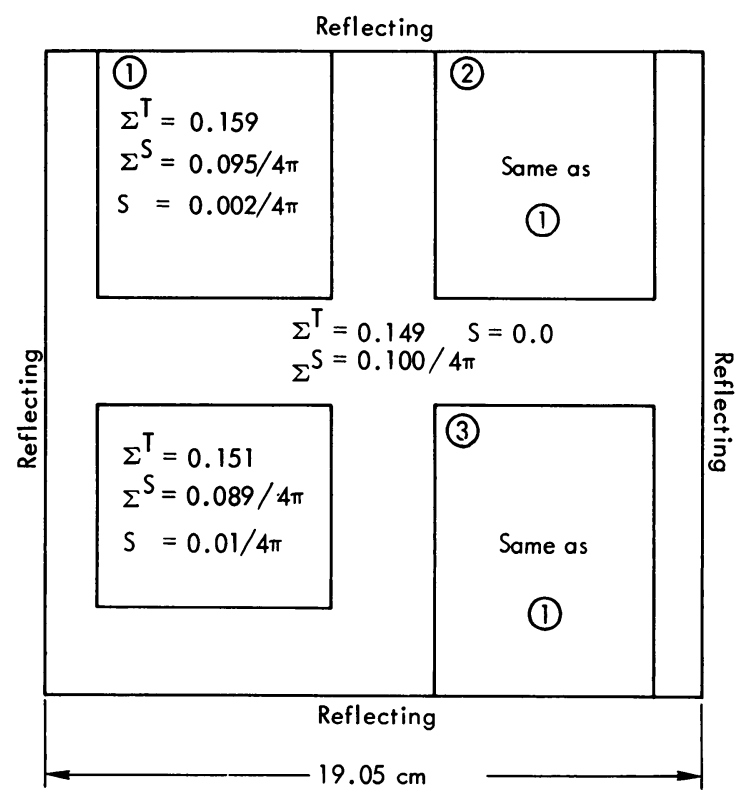

FIGURE 5 
TABLE 1

$L_{2}$ Error Bound Results

\begin{tabular}{cccc}
\hline Mesh & $\mathrm{L}_{2}$ Error Bound & $\|\tilde{\phi}\|_{L_{2}}$ & $\begin{array}{c}\text { Approximate } \\
\text { Relative Error }\end{array}$ \\
\hline Problem 1 & & & \\
$24 \times 24$ & 0.052 & 0.199 & $26 \%$ \\
$48 \times 48$ & 0.036 & 0.199 & $18 \%$ \\
$96 \times 96$ & 0.026 & 0.199 & $13 \%$ \\
$192 \times 192$ & 0.018 & 0.199 & $9 \%$ \\
& & & \\
Problem 2 & & & \\
$24 \times 24$ & 0.201 & 0.700 & $29 \%$ \\
$48 \times 48$ & 0.118 & 0.698 & $17 \%$ \\
$96 \times 96$ & 0.074 & 0.698 & $11 \%$ \\
$192 \times 192$ & 0.049 & 0.698 & $7 \%$ \\
& & & \\
Problem 3 & & & $28 \%$ \\
$44 \times 46$ & 0.075 & 0.271 & $19 \%$ \\
$88 \times 92$ & 0.053 & 0.271 & $14 \%$ \\
$176 \times 184$ & 0.037 & 0.271 & \\
\hline
\end{tabular}

TABLE 2

$L_{1}$ Error Bound Results

\begin{tabular}{cccc}
\hline Mesh & $\mathrm{L}_{1}$ Error Bound & \|\|$_{\mathrm{L}_{1}}$ & $\begin{array}{c}\text { Approximate } \\
\text { Relative Error }\end{array}$ \\
\hline Problem 1 & & & \\
$24 \times 24$ & 0.22 & 0.72 & $31 \%$ \\
$48 \times 48$ & 0.15 & 0.72 & $21 \%$ \\
$96 \times 96$ & 0.11 & 0.72 & $15 \%$ \\
$192 \times 192$ & 0.07 & 0.72 & $10 \%$ \\
& & & \\
Problem 2 & & 4.9 & $87 \%$ \\
$24 \times 24$ & 4.27 & 4.9 & $51 \%$ \\
$48 \times 48$ & 2.50 & 4.9 & $32 \%$ \\
$96 \times 96$ & 1.56 & 4.9 & $17 \%$ \\
$192 \times 192$ & 0.85 & & \\
Problem 3 & & 16.7 & $31 \%$ \\
$44 \times 46$ & 5.1 & 16.7 & $21 \%$ \\
$88 \times 92$ & 3.5 & 16.7 & $15 \%$ \\
$176 \times 184$ & 2.5 & & \\
\hline
\end{tabular}


results in Table 2. The bounds have been computed for various mesh sizes to show the effect mesh spacing has on the bounds. All of the problems used an $S_{8}$ discrete ordinates quadrature. To obtain an estimate of the relative size of the bounds, the $L_{2}$ and $L_{1}$ norms of $\phi$ have been computed and are also presented.

531 Zircon Way

Livermore, California 94550

1. J. Arkuszewski, T. KulikowsKa \& J. MiKa, "Effect of singularities on approximation in $S_{N}$ methods," Nuclear Sci. Engr., v. 49, 1972, pp. 20-26.

2. B. Davison \& J. B. SykeS, Neutron Transport Theory, Clarendon Press, Oxford, 1957. MR 20 \#2217.

3. R. B. KeLlOGG, "On the spectrum of an operator associated with the neutron transport equation," SIAM J. Appl. Math., v. 17, 1969, pp. 162-171. MR 41 \#6425.

4. N. K. MADSEN, Convergence of Difference Methods for the Linear Transport Equation, Doctoral Dissertation, University of Maryland, College Park, Maryland, 1969.

5. M. NATELSON, "A strategy for the application of space-angle synthesis to practical problems in neutron transport," Nuclear Sci.. Engr., v. 31, 1968, pp. 325-336.

6. V. S. Vladimirov, Mathematical Problems in the One-Velocity Theory of Particle Transport, AECL-1661, Atomic Energy of Canada, Ltd., Chalk River, Ontario, 1963. 